\title{
CONTROL OF THE WAVE EQUATION BY TIME-DEPENDENT COEFFICIENT
}

\author{
Antonin Chambolle $^{1}$ and Fadil Santosa ${ }^{2}$
}

\begin{abstract}
We study an initial boundary-value problem for a wave equation with time-dependent sound speed. In the control problem, we wish to determine a sound-speed function which damps the vibration of the system. We consider the case where the sound speed can take on only two values, and propose a simple control law. We show that if the number of modes in the vibration is finite, and none of the eigenfrequencies are repeated, the proposed control law does lead to energy decay. We illustrate the rich behavior of this problem in numerical examples.
\end{abstract}

Mathematics Subject Classification. 35K35, 35B37, 49J15, 49J20.

Received January 14, 2002. Revised February 15, 2002.

\section{INTRODUCTION}

The problem considered in this work is motivated by recent developments in the area of smart materials. The properties of these materials can be changed by the application of external fields, such as electrical, magnetic, or temperature. When external fields are applied, the material goes through what is known as a phase transformation. There are magnetostrictive materials whose stiffness can be altered by what is referred to as the $\Delta E$ effect [7].

A structure made with such a material, together with a sensing system that is capable of measuring deformation in the material, is considered. The control problem consists of eliminating a transient disturbance in the structure by varying the material property in response to the deformation.

In this work, we consider a simple model problem with the attributes of the more complicated structural control problem described above. The model dynamics is governed by a scalar wave equation. The control variable is the sound speed in the medium, which is assumed to take on only two values. We propose a simple control mechanism based on knowledge of the time rate of change of the potential energy in the system.

Even for this simple model problem, we found that the behavior of the problem under the proposed control law is quite rich. We begin the paper by presenting our model in the next section. Section 3 is devoted to the analysis of controlling the vibration of a single mode. While the results are of limited utility, we found the behavior of this simplified dynamics to be instructive. In Section 5 we investigate the dynamics of the full problem for existence. We show that under somewhat stringent conditions similar to [6], we are able to prove global existence. The control problem is analyzed in Section 4 for the case where there is a finite number of modes present in the initial disturbance. We establish energy decay properties under the control law. The behavior of the system, in particular, the mode mixing properties, are examined in numerical calculations.

Keywords and phrases: Control problem, time dependent wave equation, damping.

1 CEREMADE, UMR 7534 du CNRS, Université de Paris-Dauphine, 75775 Paris Cedex 16, France;

e-mail: antonin.chambolle@ceremade.dauphine.fr

2 School of Mathematics, University of Minnesota, Minneapolis, MN 55455, U.S.A.; e-mail: santosa@math.umn.edu 
Finally we note that the problem considered here is different from the control of structures by a system of smart material sensors and actuators. The smart material can be piezo-electric, in which case, the governing equations consists of a coupled set of dynamic elasticity equations and electromagnetic equations. The control problem then consists of analysis of the dynamics of bi-material body made up of elastic and piezo-electric materials. The study of such problems have been explored in numerical simulations in [3]. Other problems of this type are discussed in $[2,4]$. Our problem is more similar in nature to the dynamic composite material studied by Lurié [5], although our material is much simpler, and perhaps easier to realize.

\section{Model}

We begin with the wave equation in $\Omega \in \mathbb{R}^{n}$

$$
u_{t t}=a(t) \triangle u, \quad t>0, \quad x \in \Omega
$$

where $u(x, t)$ is the disturbance at position $x$ and time $t$. The sound speed $\sqrt{a(t)}$ is assumed be a function of time. For simplicity, let $u$ satisfy Dirichlet boundary condition

$$
u(x, t)=0, \quad \text { for } x \in \partial \Omega
$$

Initial conditions for $u$ are

$$
u(x, 0)=u_{0}(x), \quad u_{t}(x, 0)=u_{1}(x)
$$

Associated with the wave equation (1a) is the energy

$$
E(t)=\int_{\Omega}\left[\frac{1}{2} u_{t}(x, t)^{2}+\frac{1}{2} a(t)|\nabla u(x, t)|^{2}\right] \mathrm{d} x \mathrm{~d} t .
$$

The definition of energy follows that of the standard case where the coefficient $a$ is not a function of time. We recognize that this definition is somewhat arbitrary because for the PDE under consideration, energy is not a conserved quantity.

The material property $a(t)$ will be the control variable for the problem. An optimal control associated with this problem is one where we consider a finite horizon $t \in[0, T]$ (or infinite horizon), and we may attempt to find $a(t)$ which minimizes, say,

$$
\int_{0}^{T} \int_{\Omega}\left[\frac{1}{2} u_{t}(x, t)^{2}+\frac{1}{2} a(t)|\nabla u(x, t)|^{2}\right] \mathrm{d} x \mathrm{~d} t .
$$

The solution will be dependent on the initial conditions $u_{0}(x)$ and $u_{1}(x)$ in $(1 \mathrm{c})$. Instead we will derive a simple control law based on integrations-by-parts.

We multiply both sides of (1a) by $u_{t}(x, t)$ and integrate over the domain $\Omega$. We obtain

$$
\frac{\mathrm{d}}{\mathrm{d} t} \int_{\Omega}\left(\frac{1}{2} u_{t}(x, t)^{2}\right) \mathrm{d} x=-a(t) \frac{\mathrm{d}}{\mathrm{d} t} \int_{\Omega}\left(\frac{1}{2}|\nabla u(x, t)|^{2}\right) \mathrm{d} x .
$$

The integral on the left-hand side is just the kinetic energy, whereas the integral on the right-hand side is a scaled potential energy. Therefore, we choose our control to be such that kinetic energy is decreasing when possible, and made to increase as slowly as possible when not.

In our ideal model, we assume that the material property $a(t)$ can be switched between two values $a_{1}$ and $a_{2}$, with $a_{1}<a_{2}$. To make sure that kinetic energy is decreasing, or at worst, increasing only at a small rate, 
we choose the control

$$
a(t)=\phi(s(t)), \quad \phi(s)=\left\{\begin{array}{lll}
a_{1} & \text { if } & s<0 \\
a_{2} & \text { if } & s>0
\end{array}\right.
$$

where

$$
s(t)=\frac{\mathrm{d}}{\mathrm{d} t} \int_{\Omega}\left(\frac{1}{2}|\nabla u(x, t)|^{2}\right) \mathrm{d} x .
$$

We see that with this choice, the left-hand side of (3) is negative with magnitude $a_{2}|s(t)|$ when $s(t)>0$, and is positive with magnitude $a_{1}|s(t)|$, which is smaller, when $s(t)<0$. It seems thus reasonable to believe that it leads to the fastest possible decay of the energy. Observe that the value of $a(t)$ is not specified when $s(t)=0$. Further experiments and the mathematical analysis show that in this case we have to let the control belong to the interval $\left[a_{1}, a_{2}\right]$ (while a strictly bang-bang control will in some cases oscillate very quickly between the two extremal values, reaching in average an intermediate value).

The remainder of the paper is devoted to determining if the control stated in (4) leads to a decay of the energy of the system. For mainly technical reasons, most results will be established with a smoother version of the control, that is, with a different choice of $\phi$ : we will consider replacing the bang-bang function (4a) with a Lipschitz $\phi$ with roughly the same behavior, that is, nondecreasing, with $\phi(s) \simeq a_{1}$ for $s \ll-1, \phi(s) \simeq a_{2}$ for $s \gg 1$, and $\phi(0)=\left(a_{1}+a_{2}\right) / 2$.

Before we proceed, we note that formally we can pose the problem at hand as a control problem for an infinite system of ordinary differential equations by using modal expansion. Let us write

$$
u(x, t)=\sum_{n=1}^{\infty} c_{n}(t) v_{n}(x)
$$

where $v_{n}(x)$ are normalized eigenfunctions associated with eigenvalue $\omega_{n}^{2}$. Thus, we have

$$
\triangle v_{n}+\omega_{n}^{2} v_{n}=0, \quad x \in \Omega, \quad v_{n}(x)=0 \text { for } x \in \partial \Omega .
$$

Then, the $c_{n}(t)$ satisfy

$$
\ddot{c}_{n}+a(t) \omega_{n}^{2} c_{n}=0, \quad n=1,2, \cdots
$$

The initial conditions for $c_{n}$ are

$$
c_{n}(0)=c_{0 n}, \quad \dot{c}_{n}(0)=c_{1 n} .
$$

The energy associated with the system is

$$
E(t)=\frac{1}{2} \sum_{n=1}^{\infty} \dot{c}_{n}(t)^{2}+a(t) \omega_{n}^{2} c_{n}(t)^{2} .
$$

The equivalent control law for the Fourier coefficients is

$$
a(t)=\phi(s(t)), \quad s(t)=\frac{\mathrm{d}}{\mathrm{d} t} \sum_{n=1}^{\infty} \frac{\omega_{n}^{2}}{2} c_{n}(t)^{2}
$$

which can be derived directly from the infinite system of ordinary differential equations (5). 


\section{Control of one mode}

In this section, we consider the simplest case where just one mode is nonzero in (5). In this case, we can show explicitly that the bang-bang control (4a) leads to an exponential decay of the energy. We provide a natural criterion for which this control is optimal. Let the initial data in (1c) be (for simplicity)

$$
u(x, 0)=v_{n}(x), \quad u_{t}(x, 0)=0 .
$$

Then the ODE associated with the mode amplitude is

$$
\ddot{c}_{n}+a(t) \omega_{n}^{2} c_{n}=0
$$

with initial condition $c_{n}(0)=1$ and $\dot{c}_{n}(0)=0$. We use the control law $(7)$, with $\phi$ given by (4a). This leads to the bang-bang control

$$
a(t)=\left\{\begin{array}{lll}
a_{1} & \text { if } & c_{n}(t) \dot{c}_{n}(t)<0 \\
a_{2} & \text { if } \quad & c_{n}(t) \dot{c}_{n}(t)>0
\end{array}\right.
$$

The solution for constant $a$ can be given in terms of a propagator

$$
\left[\begin{array}{c}
c_{n} \\
\dot{c}_{n}
\end{array}\right](t+\delta t)=\left[\begin{array}{cc}
\cos \sqrt{a} \omega_{n} \delta t & \frac{\sin \sqrt{a} \omega_{n} \delta t}{\sqrt{a} \omega_{n}} \\
-\sqrt{a} \omega_{n} \sin \sqrt{a} \omega_{n} \delta t & \cos \sqrt{a} \omega_{n} \delta t
\end{array}\right]\left[\begin{array}{c}
c_{n} \\
\dot{c}_{n}
\end{array}\right](t)
$$

Since $a(t)$ will be piecewise constant, we will make use of this formula.

For $t>0$ and small, $\dot{c}_{n}(t)$ will be negative. Therefore, the control law $(8)$ assigns $a(t)=a_{1}$ (recall that $\left.a_{1}<a_{2}\right)$. Hence, we have

$$
c_{n}(t)=\cos \sqrt{a_{1}} \omega_{n} t, \quad \dot{c}_{n}(t)=-\sqrt{a_{1}} \omega_{n} \sin \sqrt{a_{1}} \omega_{n} t
$$

According to the control law (8), the first instance where $a(t)$ will switch to $a_{2}$ is at $T_{1}$, at which point, $c_{n}\left(T_{1}\right)=0$, and $\dot{c}_{n}\left(T_{1}\right)<0$. We easily find $T_{1}=\pi /\left(2 \sqrt{a_{1}} \omega_{n}\right)$. Thereafter, for $t>T_{1}$, we again use the propagator equation (9) with $a=a_{2}$ to find $c_{n}(t)$ and $\dot{c}_{n}(t)$. The next switch, from $a_{2}$ to $a_{1}$ occurs at $T_{2}$, where $\left(T_{2}-T_{1}\right)=\pi /\left(2 \sqrt{a_{2}} \omega_{n}\right)$. We can evaluate $c_{n}$ and $\dot{c}_{n}$ at $0, T_{1}$, and $T_{2}$, using the propagator matrix:

$$
\begin{array}{ll}
c_{n}(0)=1, & \dot{c}_{n}(0)=0 ; \\
c_{n}\left(T_{1}\right)=0, & \dot{c}_{n}(0)=-\sqrt{a_{1}} \omega_{n} ; \\
c_{n}\left(T_{2}\right)=-\sqrt{a_{1}} / \sqrt{a_{2}}, & \dot{c}_{n}(0)=0 .
\end{array}
$$

The next two times when $a(t)$ switches can be found from following the signs of $c_{n}(t)$ and $\dot{c}_{n}(t)$ and the recipe in (8). They are at $T_{3}$ and $T_{4}$ where $T_{3}=T_{1}+T_{2}$, and $T_{4}=2 T_{2}$. The values of $c_{n}$ and $\dot{c}_{n}$ at these times are

$$
\begin{array}{ll}
c_{n}\left(T_{3}\right)=0, & \dot{c}_{n}\left(T_{3}\right)=a_{1} \omega_{n} / \sqrt{a_{2}} \\
c_{n}\left(T_{4}\right)=a_{1} / a_{2}, & \dot{c}_{n}\left(T_{4}\right)=0 .
\end{array}
$$

We can calculate the energy defined by (6) and find that

$$
\begin{aligned}
& E(t)=a_{1} \frac{\omega_{n}^{2}}{2}, \quad \text { for } 0<t<T_{2}, \\
& E(t)=a_{1}^{2} \frac{\omega_{n}^{2}}{a_{2}}, \quad \text { for } T_{2}<t<T_{4} .
\end{aligned}
$$



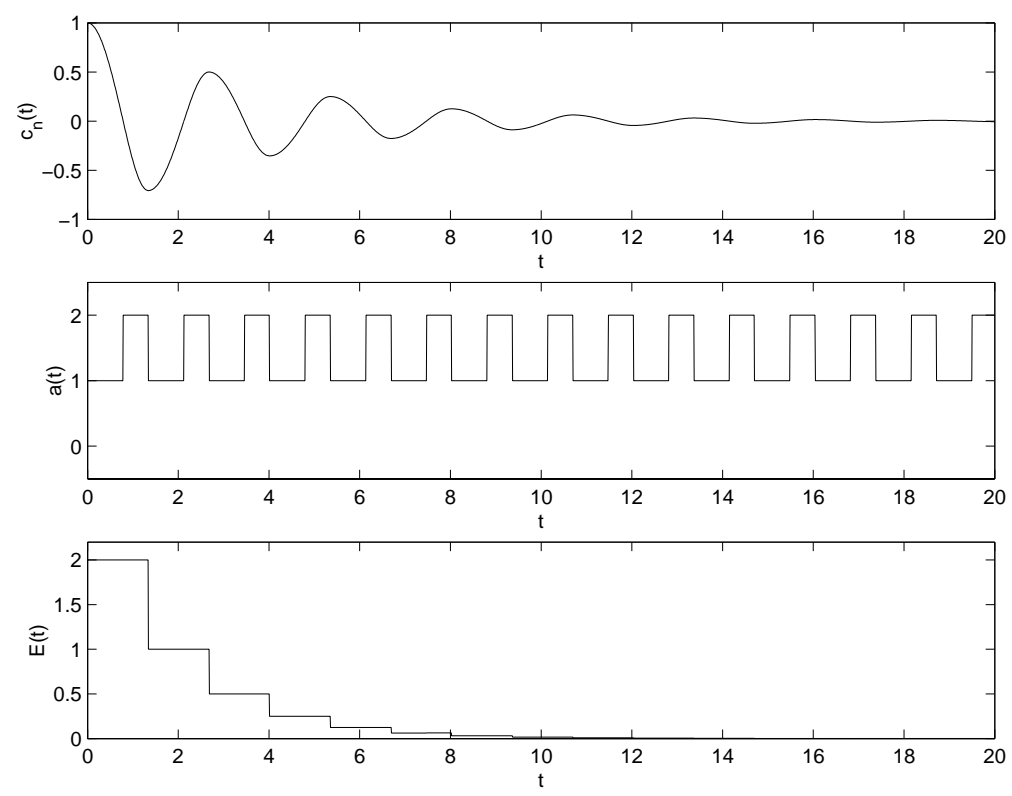

Figure 1. Energy decay for a single mode where $a_{1}=1, a_{2}=2$, and $\omega=2$. Top: Fourier coefficient $c_{n}(t)$. Center: the control parameter $a(t)$. Bottom: the energy $E(t)$.

The upshot is that by using the control law, we have reduced the initial energy by a factor of $a_{1} / a_{2}$ by the time $t=T_{2}$. Repeating this argument allows us to conclude that the energy decreases by this factor every time interval $T_{2} ;$ i.e.,

$$
E\left(k T_{2}\right)=a_{1} \omega_{n}^{2}\left(\frac{a_{1}}{a_{2}}\right)^{k}, k=1,2, \cdots
$$

We can generalize the argument to lead to the same conclusion for any initial data. Moreover, $a(t)$ is a periodic function of period $T_{2}$.

We give an illustration of the energy decay of the single mode case in Figure 1.

We next demonstrate that for the one-mode case, the control law (8) is indeed optimal for an infinite horizon control problem. Let us first rewrite the ODE as a system

$$
\dot{X}_{n}=\left[\begin{array}{cc}
0 & 1 \\
-a(t) \omega_{n}^{2} & 0
\end{array}\right] X_{n}
$$

with initial data $X_{n}(0)=x=\left(c_{n}(0), \dot{c}_{n}(0)\right)$. The the optimal control problem is the minimization

$$
J(x)=\inf _{a(.)} \int_{0}^{\infty} E(t) \mathrm{d} t
$$

where $E(t)$ defined as in $(2), E(t)=\dot{c}_{n}(t)^{2} / 2+a(t) \omega_{n}^{2} c_{n}(t)^{2} / 2$. We show the following result.

Theorem 1. The optimal control in problem (10) is given by (8).

Proof. By linearity of the equations, note that $J$ is even and homogeneous; i.e., $J(\lambda x)=\lambda^{2} J(x)$ for every $x \in \mathbb{R}^{2}$ and $\lambda \in \mathbb{R}$. 

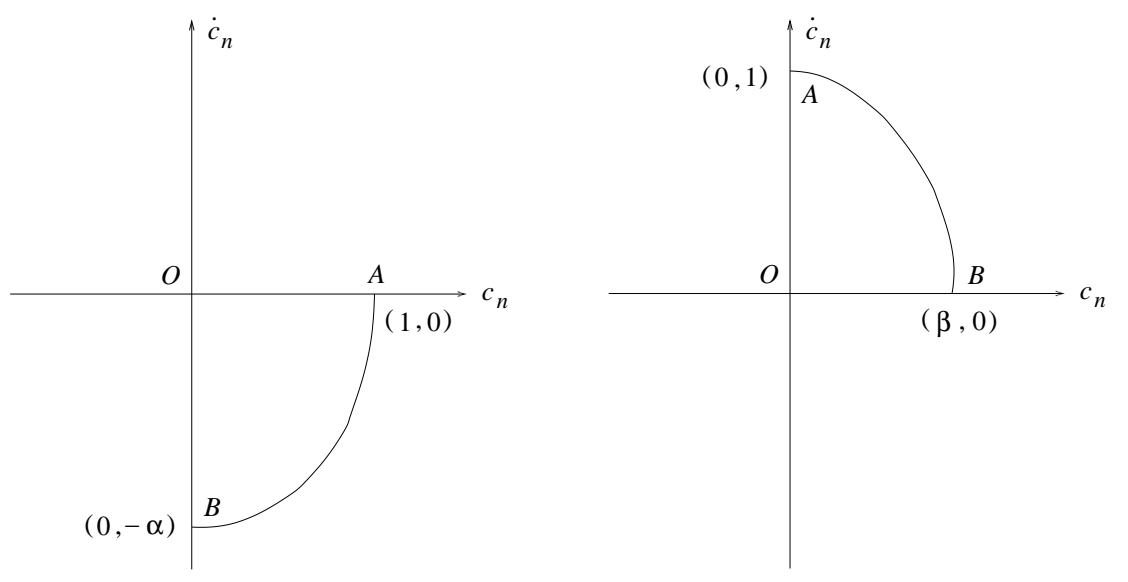

FiguRE 2. (a) The trajectory starting from $(1,0)$. (b) The trajectory starting from $(0,1)$. In both cases, optimality requires the area $A B O$ to be as small as possible.

Associated with (10) is a dynamic programming principle, that is, for every $\tau \geq 0, x \in \mathbb{R}^{2}$

$$
J(x)=\inf _{a(.)}\left\{\int_{0}^{\tau} E(t) \mathrm{d} t+J\left(X_{n}(\tau)\right)\right\} .
$$

With initial data $x=(1,0)$, in the phase plane, the trajectory starting from $x$ will live in the fourth quadrant until it reaches a value $X_{n}(\tau)=(0,-\alpha)$ at a certain "exit" time $\tau$. Thus,

$$
J((1,0))=\inf _{a(.)}\left\{\int_{0}^{\tau} E(t) \mathrm{d} t+J((0,-\alpha))\right\}
$$

or by the homogeneity and evenness of $J$,

$$
J((1,0))=\inf _{a(.)}\left\{\int_{0}^{\tau} E(t) \mathrm{d} t+\alpha^{2} J((0,1))\right\},
$$

with $c_{n}(\tau)=-\alpha$ and $\dot{c}_{n}(\tau)=0$. A sketch of the trajectory is given in Figure 2a where the starting point at $t=0$ is $A:(1,0)$, and the first exit point at $t=\tau$ is $B:(0,-\alpha)$.

Now, we study the integral $\int_{0}^{\tau} E(t) \mathrm{d} t$ where $\tau$ is the time where the trajectory reaches $B:(0,-\alpha)$. We have $X_{n}=\left(c_{n}, \dot{c}_{n}\right)$ and for $0 \leq t \leq \tau, c_{n}$ goes from 1 to 0 while $\dot{c}_{n}$ goes from 0 to $\alpha$. The integral is

$$
\int_{0}^{\tau} E(t) \mathrm{d} t=\int_{0}^{\tau} \frac{\dot{c}_{n}(t)^{2}}{2} \mathrm{~d} t+\int_{0}^{\tau} \frac{a(t) \omega_{n}^{2} c_{n}(t)^{2}}{2} \mathrm{~d} t .
$$

In the first integral we make the change of variable $s=c_{n}(t), \mathrm{d} s=\dot{c}_{n}(t) \mathrm{d} t$. It becomes $\frac{1}{2} \int_{1}^{0} \dot{c}_{n}\left(c_{n}^{-1}(s)\right) \mathrm{d} s$, which is half the area inside $A B O$.

In the second integral the change of variable is $s=\dot{c_{n}}(t)$ so that $\mathrm{d} s=-a(t) \omega_{n}^{2} c_{n}(t) \mathrm{d} t$. It becomes $-\frac{1}{2} \int_{1}^{0} c_{n}\left(\dot{c}_{n}^{-1}(s)\right) \mathrm{d} s$ and gives the same value as the first one. Thus $\int_{0}^{\tau} E(t) \mathrm{d} t$ is the area $A B O$.

At every point on the trajectory $A B$, the velocity vector is

$$
\dot{X}_{n}=\left(\dot{c}_{n},-a \omega_{n}^{2} c_{n}\right)
$$

with $c_{n}>0$, and $\dot{c}_{n}<0$. Therefore, to make the area $A B O$ as small as possible, we choose the velocity so that the second component is as small in magnitude as possible, i.e., by choosing $a=a_{1}$. With this choice, we can 
easily calculate $\tau$ and $\alpha$,

$$
\alpha=-\sqrt{a_{1}} \omega_{1}, \quad \text { and } \quad \tau=\frac{\pi}{2 \sqrt{a_{1}} \omega_{n}} .
$$

The choice $a=a_{1}$, with the resulting $\tau$, minimizes the functional in (11). We also find

$$
\int_{0}^{\tau} E(t) \mathrm{d} t=\frac{\omega_{n} \pi \sqrt{a_{1}}}{2}
$$

Thus, we obtain

$$
J((1,0))=\frac{\pi \omega_{n} \sqrt{a_{1}}}{4}+a_{1} \omega_{n}^{2} J((0,1))
$$

We proceed by computing $J((0,1))$ with the same method,

$$
J((0,1))=\inf _{a(.)}\left\{\int_{0}^{\tau} E(t) \mathrm{d} t+J((\beta, 0))\right\} .
$$

For this case, the starting point in the trajectory is $X_{n}(0)=(0,1)$, and the trajectory is in the first quadrant (see Fig. 2b). The exit time $\tau$ is the first time where $\dot{c}_{n}$ vanishes, i.e., at point $B$. Along path $A B$, both $c_{n}$ and $\dot{c}_{n}$ are positive. Again, $J((0,-\beta))=\beta^{2} J((0,1))$, and the same study shows that the optimal path minimizes the area $A B O$, obtained by choosing $a \equiv a_{2}$.

Further calculation reveals that $\beta=1 /\left(\sqrt{a_{1}} \omega_{n}\right)$, and $\tau=\pi /\left(2 \sqrt{a_{2}} \omega_{n}\right)$, leading to $\int_{0}^{\tau} E(t) \mathrm{d} t=\sqrt{a_{2}} \omega_{n} \pi / 4$. We conclude that

$$
J((0,1))=\frac{\pi \omega_{n} \sqrt{a_{2}}}{4}+\frac{1}{a_{2} \omega_{n}^{2}} J((0,1)) .
$$

We can solve for $J((1,0))$ from $(12)$ and $(13)$

$$
J((1,0))=\frac{\pi \omega_{n} \sqrt{a_{1}}}{4\left(1-a_{1} / a_{2}\right)}\left(1+\sqrt{a_{1} a_{2}} \omega_{n}^{2}\right) .
$$

The energy is finite, and our construction shows how to assign $a(t)$ to obtain the optimal control. Notice that the exit time for initial data coincides with the time $T_{1}$, and the exit time for initial data coincides with the time $T_{2}-T_{1}$ at the beginning of this section. From this, we can see that the assignment of $a(t)$ is $i d e n t i c a l$ to that in the beginning of the section. Thus we can conclude that for the one-mode case, the control law we introduced in (8) is optimal. Moreover, although we have only considered the initial value $\left(c_{n}(0), \dot{c}_{n}(0)\right)=(1,0)$, the dynamic programming principle shows that in fact our control law is optimal independently of the initial value $x$, for minimizing the criterion (10).

\section{Control of multimodal vibration}

While it is satisfying to know that the bang-bang version of the control law is also the optimal control for an infinite horizon problem in the case of one mode, it is not at all clear that the control even leads to damping when there are more modes. In the case of multiple modes, mode mixing makes any kind of explicit analysis impractical. We resort to an energy bound, and show that the bound goes to zero as $t \rightarrow \infty$, provided that $\omega_{n}$ are all distinct. In doing so rigorously, we assumed that the initial disturbance consists of only a finite number of modes. The proof is given in Section 6, and we discuss the obstruction preventing the proof of the same result in the case of infinite modes in the final discussion section. The present section is devoted to the derivation of the energy bound and numerical experimentation with the control. 


\subsection{An energy bound}

In this section, we will again consider the bang-bang control for the case of multimodal vibration. An energy bound, derived for this problem, will later be used to demonstrate damping of the system. The purpose of this section is to provide some insight into the question of damping, with details of the proof for damping deferred to Section 6 .

We begin by defining the kinetic energy and a measure of the potential energy

$$
E_{K}(t)=\sum_{n=1}^{\infty} \frac{\dot{c}_{n}^{2}}{2} \quad \text { and } \quad \hat{E}_{P}=\sum_{n=1}^{\infty} \omega_{n}^{2} \frac{c_{n}^{2}}{2} .
$$

We use the function (4a)

$$
\phi(s)=\left\{\begin{array}{lll}
a_{1} & \text { if } & s<0 \\
a_{2} & \text { if } & s>0
\end{array}\right.
$$

to define the control law. The governing equations for the Fourier coefficients $c_{n}(t)$ are

$$
\ddot{c}_{n}+\phi\left(\sum_{n=1}^{\infty} \omega_{n}^{2} c_{n} \dot{c}_{n}\right) \omega_{n}^{2} c_{n}=0, \quad n=1,2, \cdots
$$

subject to initial conditions. Identifying the argument of $\phi(\cdot)$ with $\frac{\mathrm{d}}{\mathrm{d} t} \hat{E}_{P}$, we see that

$$
\ddot{c}_{n}+\phi\left(\frac{\mathrm{d}}{\mathrm{d} t} \hat{E}_{P}\right) \omega_{n}^{2} c_{n}=0
$$

We choose $\alpha$ to be the mean

$$
\alpha=\frac{a_{1}+a_{2}}{2}
$$

and define a kind of energy

$$
E_{\alpha}(t)=E_{K}(t)+\alpha \hat{E}_{P}(t)
$$

We observe that

$$
\begin{aligned}
\frac{\mathrm{d}}{\mathrm{d} t} E_{\alpha} & =\frac{\mathrm{d}}{\mathrm{d} t} E_{K}+\alpha \frac{\mathrm{d}}{\mathrm{d} t} \hat{E}_{P} \\
& =\sum_{n=0}^{\infty} \dot{c}_{n} \ddot{c}_{n}+\alpha \omega_{n}^{2} c_{n} \dot{c}_{n} \\
& =\left[\alpha-\phi\left(\frac{\mathrm{d}}{\mathrm{d} t} \hat{E}_{P}\right)\right] \frac{\mathrm{d}}{\mathrm{d} t} \hat{E}_{P}
\end{aligned}
$$

after using (15) and (14). If $\frac{\mathrm{d}}{\mathrm{d} t} \hat{E}_{P}<0, \phi=a_{1}$, so that $\frac{\mathrm{d}}{\mathrm{d} t} E_{\alpha}(t)<0$. If on the other hand $\frac{\mathrm{d}}{\mathrm{d} t} \hat{E}_{P}>0, \phi=a_{2}$, and in that case, we still have $\frac{\mathrm{d}}{\mathrm{d} t} E_{\alpha}(t)<0$. Therefore, we conclude that

$$
\frac{\mathrm{d}}{\mathrm{d} t} E_{\alpha} \leq 0
$$


When $a(t)=a_{1}$, we can bound $E(t)$ from below by considering, for $K>0$,

$$
K E_{\alpha}(t)=\sum_{n=1}^{\infty} \frac{K}{2} \dot{c}_{n}^{2}+\frac{K}{2}\left(\frac{a_{1}+a_{2}}{2}\right) \omega_{n}^{2} c_{n}^{2} .
$$

Choosing $K=2 a_{1} /\left(a_{1}+a_{2}\right)<1$ gives us

$$
\frac{2 a_{1}}{a_{1}+a_{2}} E_{\alpha}(t) \leq E(t)
$$

A similar argument gives an upper bound. The energy (6) therefore satisfies a global bound

$$
\frac{2 a_{1}}{a_{1}+a_{2}} E_{\alpha}(t) \leq E(t) \leq \frac{2 a_{2}}{a_{1}+a_{2}} E_{\alpha}(t) .
$$

The results in (17) and (18) are the ingredients needed to establish that the system is damped. The latter imply that the energy in the system is bounded above, and below, by a nonincreasing "weighted" energy $E_{\alpha}(t)$. It remains to be shown that the only limit of $E_{\alpha}(t)$ is zero. We are indeed able to demonstrate this fact when there are a finite number of modes present in the vibration, and the frequencies $\omega_{n}$ are distinct. We defer demonstration of this fact to Section 6, after we have established well-posedness of the initial value problem in Section 5. In establishing the result, we replace the function $\phi(\cdot)$ in $(15)$ with a Lipschitz function. In the next subsection, we will study the behavior of the damping in numerical experiments.

\subsection{Numerical examples}

In order to obtain detail behavior of the wave equation with the control law that we proposed, we consider the discrete dynamical system given by (5) with time-dependent coefficients given by (7). We assume that we have a finite number of modes with frequencies

$$
\omega_{n}=n \pi, \quad n=1,2, \cdots, N
$$

Note that we do not have repeated eigenvalues since as we will show later, the control law we prescribed does not work when there are repeated eigenvalues. The material properties are chosen to be $a_{1}=1$, and $a_{2}=4$. The latter chosen large in order to have large damping. The differential equation is first rewritten as a first order system. In all our examples, we take $N \leq 30$, but to capture the dynamics accurately, we take small time increments $\Delta t=2 /\left(100 \times 30 \sqrt{a_{2}}\right)$. That is, if $N=30$, the shortest period in time is sampled at 100 points. Within this time increment, $a(t)$ is assumed to be constant. With $a(t)$ piecewise constant, we can use the propagator method (9) to evolve the dynamics, using the control law (7) to choose $a(t)$.

It must be pointed out here that the control of assigning

$$
a=a_{1} \text { if } \frac{\mathrm{d}}{\mathrm{d} t} \hat{E}_{P}<0, \text { and } a=a_{2} \text { if } \frac{\mathrm{d}}{\mathrm{d} t} \hat{E}_{P}>0
$$

leaves the choice of $a$ ambiguous when $\frac{\mathrm{d}}{\mathrm{d} t} \hat{E}_{P}=0$; we will simply say that $a \in\left[a_{1}, a_{2}\right]$ when this happens. As we shall see shortly, this turns out to have interesting consequences. The fact is that $\frac{\mathrm{d}}{\mathrm{d} t} \hat{E}_{P}$ can be very small. If it is small and positive, then $a_{2}$ is assigned, leading to a small and negative $\frac{\mathrm{d}}{\mathrm{d} t} \hat{E}_{P}$ in the next time increment. Then $a_{1}$ is assigned, leading to $\frac{\mathrm{d}}{\mathrm{d} t} \hat{E}_{P}$ small and positive in the next time increment. Thus $a(t)$ oscillates while at the same time $\hat{E}_{P}$ is nearly constant over a time interval. This oscillation will be more rapid as we take smaller time samples - an averaging phenomenon. The system is attempting to take choose a value for $a(t)$ which is in the interval $\left[a_{1}, a_{2}\right]$ by rapid oscillation. In this sense, the calculations must be interpreted as an 

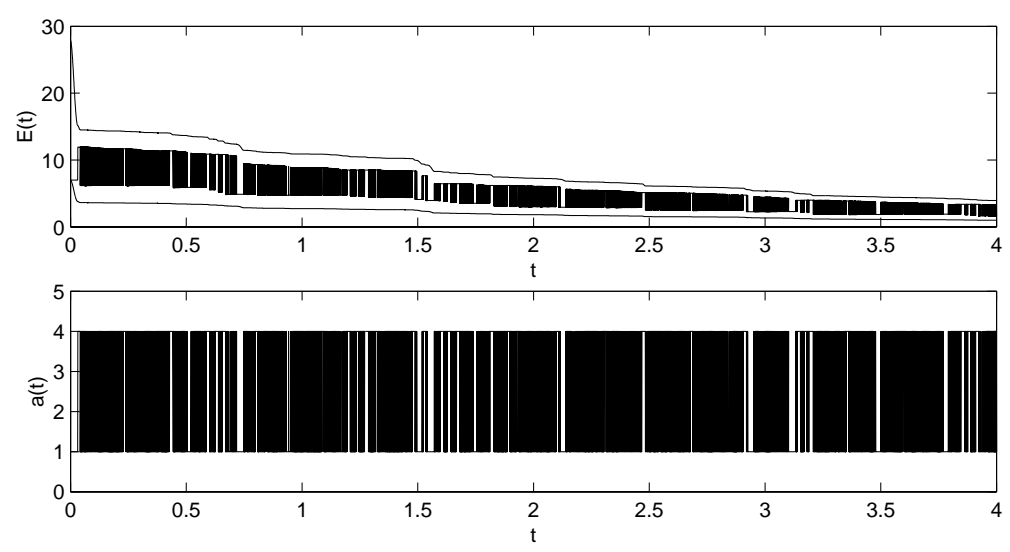

Figure 3. Random initial data with 20 modes. Top: energy vs. time. Overplotted are the upper and lower bounds in (18). Bottom: control coefficient vs. time. Black out regions correspond to rapid oscillations.

approximation of the continuum problem. The limit behavior of $a(t)$ as the time increment goes to zero is some kind of local average of the rapidly oscillating solution.

In all the examples, the initial velocity of each mode is set to zero; i.e., $\dot{c}_{n}(0)=0$.

Example 1. In the first example, we take $N=20$, and set $c_{n}(0)=p / n$ where $p$ is a random number in the interval $[-0.5,0.5]$. Figure 4 (top) shows the decay in the energy $E(t)$. In the solid black parts of the curve, the energy is rapidly oscillating. Shown also are the upper and lower bounds for the problem as predicted by (18). We also display the control $a(t)$ in Figure 4 (bottom). Again we have rapid oscillation in $a(t)$ which reflects the system's attempt to achieve $a$ between $a_{1}$ and $a_{2}$ by rapid oscillation.

Example 2. We choose $N=5$ with $c_{n}(0)$ equal to zero except for $c_{1}(0)=c_{5}(0)=1$. The example is designed to show the damping mechanism. The system is evolved over the time interval $[0,4]$. In Figure 5 (top) we show the energy decay as a function of $t$. The corresponding time-dependent coefficient $a(t)$ is shown in Figure 5 (middle). Note also the self-similar nature of the coefficient and the energy plots. Plots of the coefficients $c_{1}(t)$ and $c_{5}(t)$ are given in Figure 5 (bottom). It can be seen that the higher frequency disturbance is damped more quickly than the low frequency component.

Example 3. In this example, we investigate the behavior of the system to smoothing of the control law. The initial data is $c_{n}(0)=1 / n$, for $n=1, \cdots, 30$. First we solve the problem with control law $(7)$. Next, the problem is solved with control law

$$
a=\phi\left(\frac{\mathrm{d}}{\mathrm{d} t} \sum \omega_{n}^{2} c_{n}^{2}\right), \quad \phi(s)=\frac{1}{2}\left[\left(a_{1}+a_{2}\right)+\left(a_{2}-a_{1}\right) \tanh (k s)\right]
$$

with $k=0.5$ and $k=0.05$. The control law (7) is the formal limit of the above as $k \rightarrow \infty$. The effect of $k$ is to smooth the transition between $a_{1}$ and $a_{2}$. What we observe is that both the coefficient $a(t)$ and the energy $E(t)$ becomes smoother (especially at later times when the high frequency information has been damped) as we make $k$ smaller. We display this behavior in Figures 5 and 6 . We believe that there is a homogenization phenomenon inherent in the process, and that a limit behavior may be possible to characterize. 

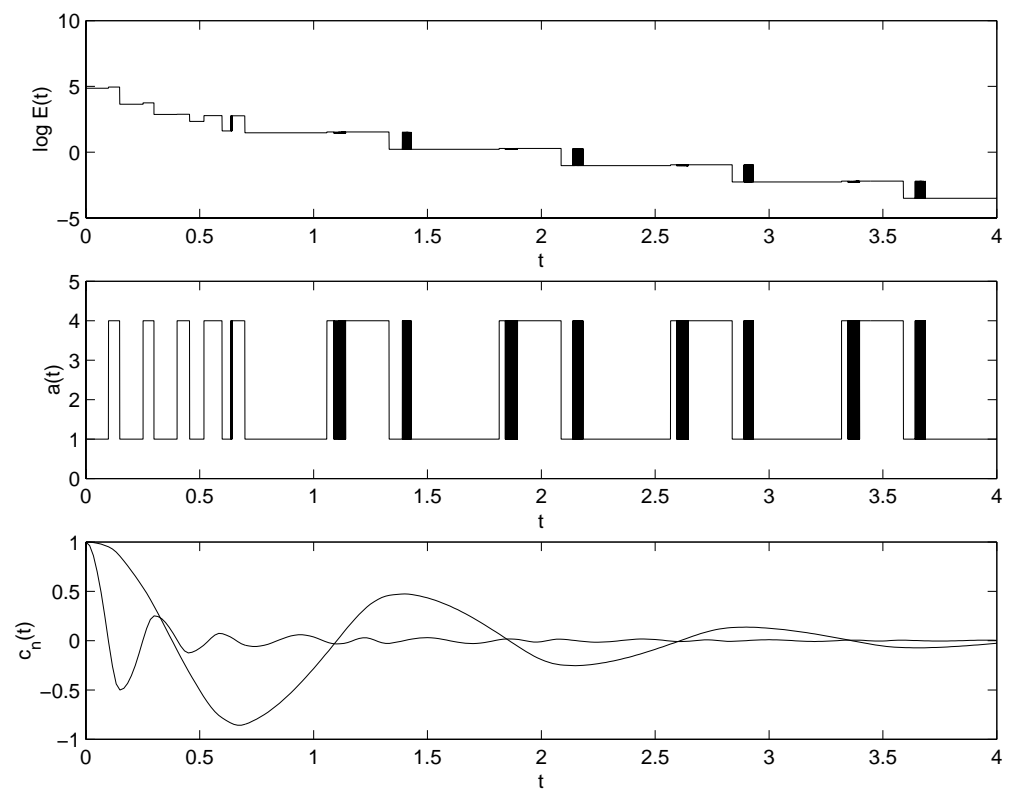

Figure 4. Demonstration of damping with 2 modes. Top: plot of $\log E(t)$. Middle: plot of $a(t)$. Bottom: plot of $c_{1}(t)$ and $c_{5}(t)$. Note the self-similar nature of the control and the logarithm of the energy after some of the high frequency component has been damped.

\section{EXISTENCE OF SOLUTION}

We will study the existence of solutions for our problem in the case where the equation is of the form

$$
\ddot{c}_{n}+\phi\left(\sum_{n=1}^{\infty} \omega_{n}^{2} c_{n} \dot{c}_{n}\right) \omega_{n}^{2} c_{n}=0, \quad n=1,2, \cdots,
$$

with given initial values $c_{n}(0), \dot{c}_{n}(0), n \geq 1$, but now (as in Example 3 above) $\phi$ is a nondecreasing Lipschitzcontinuous function, such that

$$
\lim _{s \rightarrow-\infty} \phi(s)=a_{1}, \quad \lim _{s \rightarrow+\infty} \phi(s)=a_{2}, \quad \text { and } \phi^{-1}(\alpha)=0,
$$

where $\alpha=\left(a_{1}+a_{2}\right) / 2$. We denote by $L$ the Lipschitz constant of $\phi(\cdot)-0 \leq \phi^{\prime} \leq L$.

\subsection{Finite number of modes}

We consider first the case where all but a finite number of modes are zero. For simplicity we can consider just the first $N$ modes $(N \geq 1)$ of the system. In this case, the existence of a solution is obvious, and given by the Cauchy-Lipschitz theorem. We let

$$
X=\left(\begin{array}{c}
X_{1} \\
\vdots \\
X_{N}
\end{array}\right)
$$



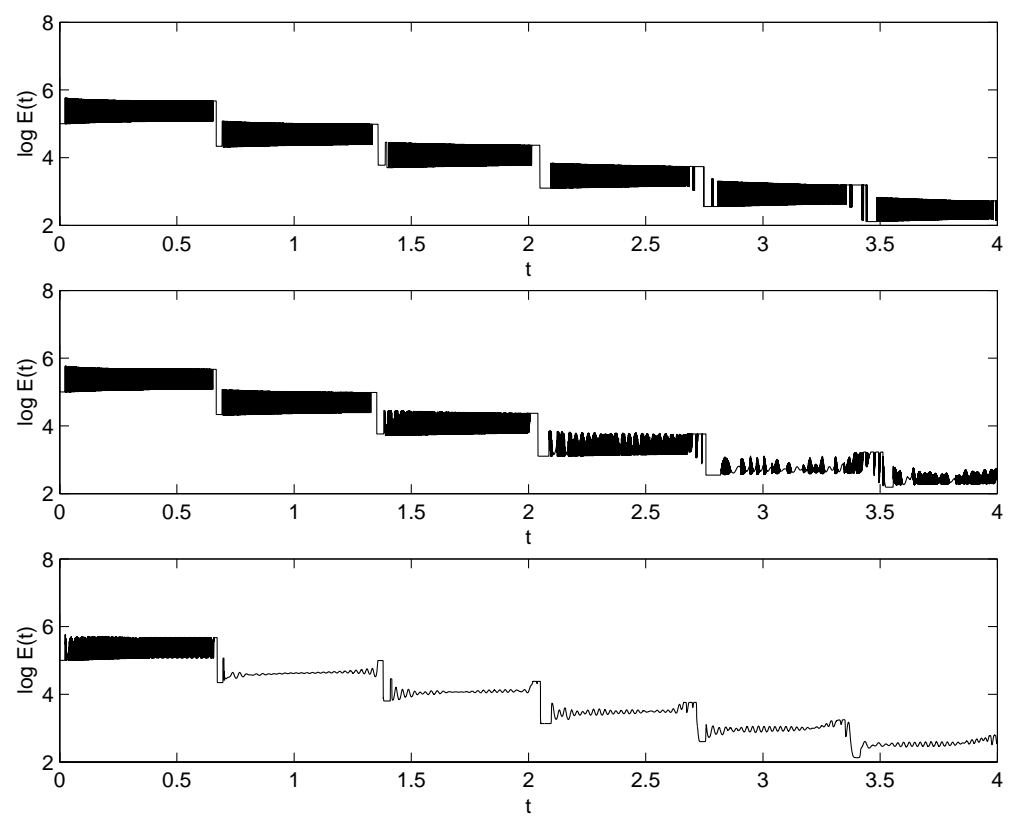

FigurE 5. The effect of smoothing the control law: plots of $\log E(t)$. The control $a=\phi\left(\frac{\mathrm{d}}{\mathrm{d} t} \hat{E}_{P}\right)$, see (14). Top: $\phi(s)$ is binary: $a_{1}$ or $a_{2}$. Middle and bottom: $\phi(s)$ is a smooth, and smoother, function of $s$.

where for every $n=1, \ldots, N$,

$$
X_{n}=\left(\begin{array}{c}
\omega_{n} c_{n} \\
\dot{c}_{n}
\end{array}\right)
$$

Then, the equation is $\dot{X}=F(X)$ where the transformation $F(X)$ multiplies the component $X_{n}$ of the vector $X$ by the square matrix

$$
\omega_{n}\left(\begin{array}{cc}
0 & 1 \\
-\phi\left(\sum_{m=1}^{N} \omega_{m}^{2} c_{m} \dot{c}_{m}\right) & 0
\end{array}\right)
$$

It is clear that $F$ is locally Lipschitz-continuous, hence for every initial data $X^{0}$ associated to a set of initial values $c_{1}(0), \dot{c}_{1}(0), \ldots, c_{N}(0), \dot{c}_{N}(0)$, there exist a maximal interval $[0, T) \subseteq[0,+\infty)$ and a solution $X(t)$ satisfying the equation on $[0, T)$, with $X(0)=X^{0}$. Notice that this solution is also unique.

In order to show that $T=+\infty$ it is enough to show that the solution $X(t)$ remains bounded for every finite $t$. Clearly, $\|F(X)\| \leq \omega_{N}\left(1 \vee a_{2}\right)\|X\|$ for every $X \in \mathbb{R}^{2 N}$. Hence by Gronwall's lemma $\|X(t)\| \leq \exp (k t)\left\|X_{0}\right\|$, with $k=\omega_{N}\left(1 \vee a_{2}\right)$, showing that $T=+\infty$. In fact, for every $n \geq 1$ and $t \geq 0$ we have

$$
\dot{c}_{n}(t)^{2}+\omega_{n}^{2} c_{n}(t)^{2} \leq \mathrm{e}^{2 \omega_{n}\left(1 \vee a_{2}\right) t}\left(\dot{c}_{n}(0)^{2}+\omega_{n}^{2} c_{n}(0)^{2}\right) .
$$

\subsection{Infinite number of modes}

If the number of modes is infinite, the existence of a solution is less straightforward. We can show two different results:

- an existence result for small time, for an initial data with some regularity; 

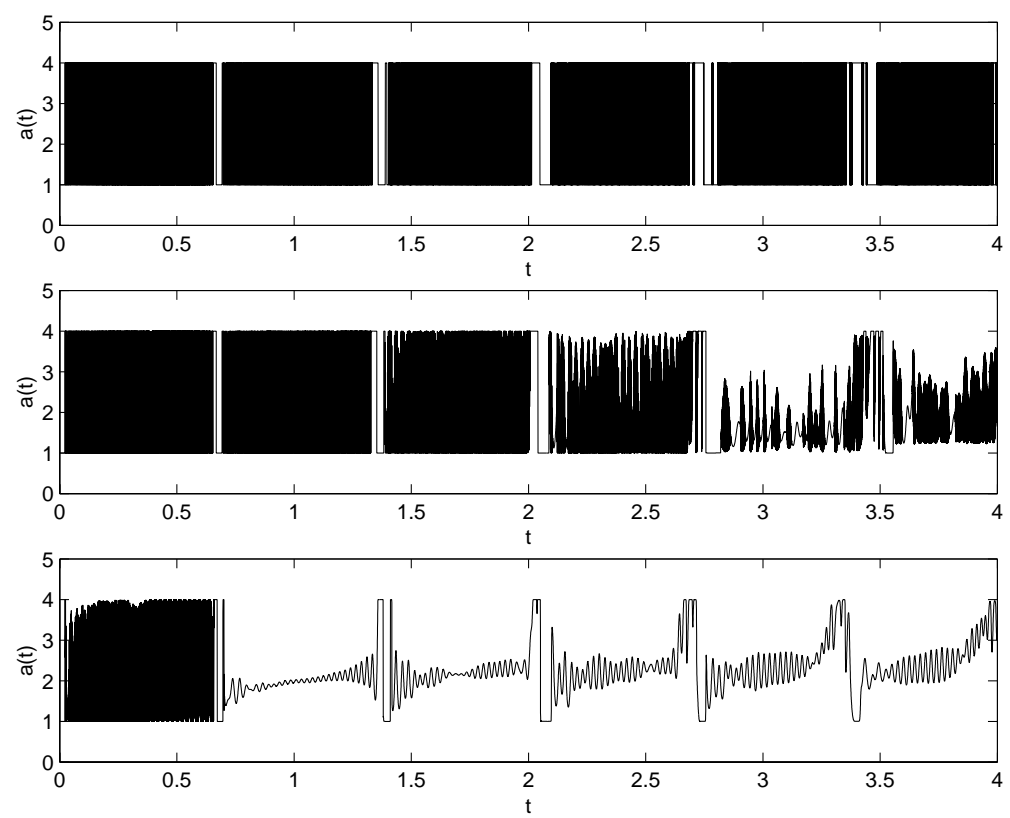

Figure 6. The effect of smoothing the control law: plots of $a(t)$. Top: $\phi(s)$ is binary: $a_{1}$ or $a_{2}$. Middle and bottom: $\phi(s)$ is a smooth, and smoother, function of $s$.

- an existence result for all times, but requiring very strong regularity hypothesis on the initial data $u_{0}(x)$ and $u_{1}(x)$, requiring them to be analytic.

The proof is based on a Galerkin approximation method, as in [6] (see also [1]). We consider a set of initial data $\left(c_{n}(0), \dot{c}_{n}(0)\right)_{n=1}^{\infty}$. For every $N \geq 1$, we denote by $\left(c_{n}^{N}(t)\right)_{n=1}^{N}$ the solution with $N$ modes corresponding to the initial data $\left(c_{n}(0), \dot{c}_{n}(0)\right)_{n=1}^{N}$. For every $N$, let

$$
a^{N}(t)=\phi\left(\sum_{m=1}^{N} \omega_{m}^{2} c_{m}^{N} \dot{c}_{m}^{N}\right)
$$

and for every $k \geq 0$ let

$$
\mathcal{F}_{k}^{N}(t)=\sum_{n=1}^{N} \omega_{n}^{2 k}\left(\dot{c}_{n}^{N}(t)^{2}+a^{N}(t) \omega_{n}^{2} c_{n}^{N}(t)^{2}\right)
$$

We also let

$$
\overline{\mathcal{F}}_{k}=\sum_{n=1}^{\infty} \omega_{n}^{2 k}\left(\dot{c}_{n}(0)^{2}+a_{2} \omega_{n}^{2} c_{n}(0)^{2}\right) .
$$

Our first result is the following:

Theorem 2. Assume $\overline{\mathcal{F}}_{1}<+\infty$. Then there exists a time $T^{*} \geq\left(L \overline{\mathcal{F}}_{1}\right)^{-1}$, and a solution of $(19)$ on $\left[0, T^{*}\right)$. Moreover, the coefficient $\phi\left(\sum_{n=1}^{\infty} \omega_{n}^{2} c_{n} \dot{c}_{n}\right)$ of the wave equation is continuous in time on $\left[0, T^{*}\right)$. 
Proof. First, we differentiate $\mathcal{F}_{k}^{N}(t)$,

$$
\frac{\mathrm{d} \mathcal{F}_{k}^{N}}{\mathrm{~d} t}=\sum_{n=1}^{N} \omega_{n}^{2 k}\left(2 \ddot{c}_{n}^{N} \dot{c}_{n}^{N}+2 a^{N} \omega_{n}^{2} \dot{c}_{n}^{N} c_{n}^{N}+\dot{a}^{N} \omega_{n}^{2}\left(c_{n}^{N}\right)^{2}\right) .
$$

Since $\ddot{c}_{n}^{N}=-a^{N} \omega_{n}^{2} c_{n}^{N}$, the expression simplifies to

$$
\frac{\mathrm{d} \mathcal{F}_{k}^{N}}{\mathrm{~d} t}=\sum_{n=1}^{N} \dot{a}^{N} \omega_{n}^{2 k+2}\left(c_{n}^{N}\right)^{2}
$$

We proceed by differentiating $a^{N}(t)$ in (22)

$$
\begin{aligned}
\dot{a}^{N} & =\phi^{\prime}\left(\sum_{m=1}^{N} \omega_{m}^{2} c_{m}^{N} \dot{c}_{m}^{N}\right) \sum_{n=1}^{N} \omega_{n}^{2}\left(\left(\dot{c}_{n}^{N}\right)^{2}+c_{n}^{N} \ddot{c}_{n}^{N}\right) \\
& =\phi^{\prime}\left(\sum_{m=1}^{N} \omega_{m}^{2} c_{m}^{N} \dot{c}_{m}^{N}\right) \sum_{n=1}^{N} \omega_{n}^{2}\left(\left(\dot{c}_{n}^{N}\right)^{2}-a^{N} \omega_{n}^{2}\left(c_{n}^{N}\right)^{2}\right)
\end{aligned}
$$

We deduce that for every $k \geq 0$,

$$
\frac{\mathrm{d} \mathcal{F}_{k}^{N}}{\mathrm{~d} t}(t) \leq L \mathcal{F}_{1}^{N}(t) \mathcal{F}_{k}^{N}(t)
$$

where $L$ is the Lipschitz constant of $\phi$.

First assume that $\overline{\mathcal{F}}_{1}<+\infty$. For every $N$, we have $\mathcal{F}_{1}^{N}(0) \leq \overline{\mathcal{F}}_{1}$. Choosing $k=1$ in $(23)$ we get that $\dot{\mathcal{F}}_{1}^{N} \leq L\left(\mathcal{F}_{1}^{N}\right)^{2}$, hence

$$
\mathcal{F}_{1}^{N}(t) \leq \frac{\mathcal{F}_{1}^{N}(0)}{1-L \mathcal{F}_{1}^{N}(0) t}
$$

as long as $t<\left(L \mathcal{F}_{1}^{N}(0)\right)^{-1}$. In particular, if $t<T^{*}=\left(L \overline{\mathcal{F}}_{1}\right)^{-1}$, we see that

$$
\mathcal{F}_{1}^{N}(t) \leq \frac{\overline{\mathcal{F}}_{1}}{1-L \overline{\mathcal{F}}_{1} t}<+\infty
$$

for every $N \geq 1$.

We want now to send $N$ to infinity. We first notice that there exists a subsequence of $\left(a^{N}\right)_{N \geq 1}$, that we will still denote by $a^{N}$, and a function $a \in L^{\infty}\left([0,+\infty),\left[a_{1}, a_{2}\right]\right)$ such that $a^{N}$ goes to $a$ weakly-* in $L^{\infty}$ as $N \rightarrow+\infty$.

For a fixed $n \geq 1$, since inequality (21) is valid for $\left(c_{n}^{N}, \dot{c}_{n}^{N}\right)$ as soon as $N \geq n$, we see that $c_{n}^{N}$, $\dot{c}_{n}^{N}$, and $\ddot{c}_{n}^{N}=-\omega_{n}^{2} a^{N} c_{n}^{N}$ are bounded uniformly in $N$ on the interval $\left[0, T^{*}\right]$. Hence we may assume (possibly extracting a further subsequence) that $c_{n}^{N}$ and $\dot{c}_{n}^{N}$ converge uniformly in $\left[0, T^{*}\right]$ as $N \rightarrow \infty$ to respectively $c_{n}$ and $\dot{c}_{n}$, where $c_{n}$ is the (unique) solution of

$$
\ddot{c}_{n}(t)+\omega_{n}^{2} a(t) c_{n}(t)=0
$$

with initial values $c_{n}(0), \dot{c}_{n}(0)$.

Now, by $(24)$, we see that for every $t<T^{*}, \mathcal{F}_{1}^{N}(t)$ is bounded uniformly in $N$. Hence (letting $c_{n}^{N} \equiv 0$ whenever $n>N)$, both vectors $\left(\omega_{n} \dot{c}_{n}^{N}(t)\right)_{n=1}^{\infty}$ and $\left(\omega_{n}^{2} c_{n}^{N}(t)\right)_{n=1}^{\infty}$ are uniformly bounded in $\ell^{2}(\mathbb{N})$ as $N$ goes to 
infinity. We deduce easily (since $\omega_{n} \uparrow+\infty$ as $\left.n \uparrow+\infty\right)$ that for every $\delta>0,\left(\omega_{n}^{1-\delta} \dot{c}_{n}^{N}(t)\right)_{n=1}^{\infty}$ converges strongly in $\ell^{2}(\mathbb{N})$ to $\left(\omega_{n}^{1-\delta} \dot{c}_{n}(t)\right)_{n=1}^{\infty}$, as well as $\left(\omega_{n}^{2-\delta} c_{n}^{N}(t)\right)_{n=1}^{\infty}$ to $\left(\omega_{n}^{2-\delta} c_{n}(t)\right)_{n=1}^{\infty}$, as $N \rightarrow \infty$.

In particular, the scalar product

$$
\sum_{n=1}^{N} \omega_{n}^{2} c_{n}^{N}(t) \dot{c}_{n}^{N}(t)=\left\langle\left(\omega_{n}^{3 / 2} c_{n}^{N}\right)_{n=1}^{\infty},\left(\omega_{n}^{1 / 2} c_{n}^{N}\right)_{n=1}^{\infty}\right\rangle_{\ell^{2}}
$$

converges, as $N \rightarrow \infty$, to $\sum_{n=1}^{\infty} \omega_{n}^{2} c_{n}(t) \dot{c}_{n}(t)$, and since $\phi$ is continuous we deduce that for every $t<T^{*}$,

$$
a(t)=\phi\left(\sum_{n=1}^{\infty} \omega_{n}^{2} c_{n}(t) \dot{c}_{n}(t)\right) .
$$

This shows the existence of a solution to our problem, for $t<T^{*}=\left(L \overline{\mathcal{F}}_{1}\right)^{-1}$.

Notice furthermore that since

$$
\left|\frac{\mathrm{d}}{\mathrm{d} t}\left(\sum_{n=1}^{N} \omega_{n}^{2} c_{n}^{N} \dot{c}_{n}^{N}\right)\right|=\left|\sum_{n=1}^{N} \omega_{n}^{2}\left(\left(\dot{c}_{n}^{N}\right)^{2}-a^{N} \omega_{n}^{2}\left(c_{n}^{N}\right)^{2}\right)\right| \leq \mathcal{F}_{1}^{N},
$$

which, by (24), is uniformly bounded on every interval $[0, T], T<T^{*}$, the convergence of $\sum_{n=1}^{N} \omega_{n}^{2} c_{n}^{N} \dot{c}_{n}^{N}$ to $\sum_{n=1}^{\infty} \omega_{n}^{2} c_{n} \dot{c}_{n}$ is in fact uniform on every such interval. This shows that this function, as well as $a(t)$, is in fact continuous in time. Theorem 2 is proven.

The next result is the following:

Theorem 3. Assume $\overline{\mathcal{F}}_{0}<+\infty$, and assume that the initial data satisfies $\liminf _{k \rightarrow \infty}\left(\overline{\mathcal{F}}_{k}\right)^{1 / k} / k<+\infty$. Then there exists a solution of (19) on $[0,+\infty)$.

Proof. We essentially follow the proof of Pohozaev [6] for a similar problem. In Section 4.1, equation (16a, 16b), we introduced the energy

$$
E_{\alpha}^{N}(t)=\sum_{n=1}^{N} \frac{\dot{c}_{n}^{N}(t)^{2}}{2}+\alpha \omega_{n}^{2} \frac{c_{n}^{N}(t)^{2}}{2}
$$

We have that

$$
\frac{\mathrm{d} E_{\alpha}^{N}}{\mathrm{~d} t}(t)=\left(\alpha-a^{N}(t)\right) \sum_{n=1}^{N} \omega_{n}^{2} c_{n}^{N}(t) \dot{c}_{n}^{N}(t),
$$

and since $a^{N}=\phi\left(\sum_{n=1}^{N} \omega_{n}^{2} c_{n}^{N} \dot{c}_{n}^{N}\right)$ and $s \mapsto(\alpha-\phi(s)) s$ is always negative (except at $s=0$ where it vanishes), we deduce that $E_{\alpha}^{N}(t)$ is nonincreasing. Hence $E_{\alpha}^{N}(t) \leq E_{\alpha}^{N}(0)$ for every $t \geq 0$, and we deduce the following estimate, valid for every $N \geq 1$ and $t \geq 0$ :

$$
\mathcal{F}_{0}^{N}(t) \leq\left(\frac{a_{2}}{\alpha}\right) \overline{\mathcal{F}}_{0}
$$

Now, by Hölder's inequality,

$$
\mathcal{F}_{1}^{N}(t) \leq\left(\mathcal{F}_{0}^{N}(t)\right)^{1-\frac{1}{k}}\left(\mathcal{F}_{k}^{N}(t)\right)^{\frac{1}{k}},
$$


for every $k \geq 1$ and $t \geq 0$. Recalling (23) and (25), we find that for every $k \geq 1$ and $t \geq 0$,

$$
\frac{\mathrm{d} \mathcal{F}_{k}^{N}}{\mathrm{~d} t}(t) \leq \frac{a_{2} L}{\alpha}\left(\overline{\mathcal{F}}_{0}\right)^{1-\frac{1}{k}}\left(\mathcal{F}_{k}^{N}(t)\right)^{1+\frac{1}{k}}
$$

We assume (as is natural) that $\overline{\mathcal{F}}_{0}<+\infty$ and let $c=a_{2} L\left(1 \vee \overline{\mathcal{F}}_{0}\right) / \alpha$. Computing the time-derivative of $\left(\mathcal{F}_{k}^{N}\right)^{-1 / k}$ we deduce from $(27)$ that as long as $t<\left(c\left(\mathcal{F}_{k}^{N}(0)\right)^{1 / k} / k\right)^{-1}$,

$$
\left(\mathcal{F}_{k}^{N}(t)\right)^{\frac{1}{k}} \leq \frac{\left(\mathcal{F}_{k}^{N}(0)\right)^{\frac{1}{k}}}{1-c{\frac{\left(\mathcal{F}_{k}^{N}(0)\right)^{\frac{1}{k}}}{k}}^{k}} .
$$

Hence, for every $N \geq 1, k \geq 1$, and $t<\left(c\left(\overline{\mathcal{F}}_{k}\right)^{1 / k} / k\right)^{-1}$

$$
\left(\mathcal{F}_{k}^{N}(t)\right)^{\frac{1}{k}} \leq \frac{\left(\overline{\mathcal{F}}_{k}\right)^{\frac{1}{k}}}{1-c{\frac{\left(\overline{\mathcal{F}}_{k}\right)^{\frac{1}{k}}}{k}}^{k}} .
$$

Let us set $\tau=\limsup _{k \rightarrow \infty}\left(c\left(\overline{\mathcal{F}}_{k}\right)^{1 / k} / k\right)^{-1}$. Since we assumed the finiteness of $\liminf _{k \rightarrow \infty}\left(\overline{\mathcal{F}}_{k}\right)^{1 / k} / k$, we have $\tau>0$.

If $T<\tau$, then there exists (an arbitrarily large) $k \geq 1$ such that $T<\left(c\left(\overline{\mathcal{F}}_{k}\right)^{1 / k} / k\right)^{-1}$, hence on $[0, T]$ the energy $\mathcal{F}_{k}^{N}(t)$ is finite and uniformly bounded by $\overline{\mathcal{F}}_{k} /\left(1-c\left(\overline{\mathcal{F}}_{k}\right)^{1 / k} / k\right)^{k}$. By $(26), \mathcal{F}_{1}^{N}(t)$ is also uniformly bounded and following the proof of the previous theorem we get the existence of a solution $\left(c_{n}(t)\right)_{n \geq 1}$ of $(19)$ on the interval $[0, T]$.

The fact that we get uniform bounds on $\mathcal{F}_{k}^{N}(t)$ on $[0, T]$ for arbitrarily large values of $k$ yields the strong convergence in $\ell^{2}(\mathbb{N})$ of $\left(\omega_{n}^{1+k} c_{n}^{N}\right)_{n=1}^{\infty}$ and $\left(\omega_{n}^{k} \dot{c}_{n}^{N}\right)_{n=1}^{\infty}$ to respectively $\left(\omega_{n}^{1+k} c_{n}\right)_{n=1}^{\infty}$ and $\left(\omega_{n}^{k} \dot{c}_{n}\right)_{n=1}^{\infty}$ as $N \rightarrow \infty$, for any $k$, (uniformly on $[0, T]$ ), as well as the uniform convergence of $a^{N}$ to $a=\phi\left(\sum_{n=1}^{\infty} \omega_{n}^{2} c_{n} \dot{c}_{n}\right)$, which is thus continuous.

In addition, we get that for every $k \geq 1$,

$$
\lim _{N \rightarrow \infty} \mathcal{F}_{k}^{N}(t)=\sum_{n=1}^{\infty} \omega_{n}^{2 k}\left(\dot{c}_{n}(t)^{2}+a(t) \omega_{n}^{2} c_{n}(t)^{2}\right)<+\infty
$$

We denote this energy by $\mathcal{F}_{k}(t)$. It is a continuous function of time, and all the inequalities proven so far for the energies $\mathcal{F}_{k}^{N}$ are also valid for $\mathcal{F}_{k}$.

Now, we let $T^{*}$ be the maximal time (possibly infinite) such that for any $T<T^{*}$, the solution exists and the energies $\mathcal{F}_{k}$ are finite and continuous in time, satisfying (23). We have seen that $T^{*} \geq \tau$ and wish to show that $T^{*}=+\infty$.

If we fix $T<T^{*}$ and let $\lambda=\max _{t \in[0, T]} \mathcal{F}_{1}(t)$ we get from (23) that for every $t \in[0, T]$,

$$
\mathcal{F}_{k}(t) \leq \mathrm{e}^{\lambda L t} \mathcal{F}_{k}(0) \leq \mathrm{e}^{\lambda L t} \overline{\mathcal{F}}_{k}
$$

Letting for every $k$

$$
\overline{\mathcal{F}}_{k}{ }^{\prime}=\sum_{n=1}^{\infty} \omega_{n}^{2 k}\left(\dot{c}_{n}(T)^{2}+a_{2} \omega_{n}^{2} c_{n}(T)^{2}\right) \leq \frac{a_{2}}{a_{1}} \mathcal{F}_{k}(T)
$$


we get

$$
\frac{\left(\overline{\mathcal{F}}_{k}^{\prime}\right)^{\frac{1}{k}}}{k} \leq\left(\frac{a_{2}}{a_{1}}\right)^{\frac{1}{k}} \mathrm{e}^{\frac{\lambda L T}{k}} \frac{\left(\overline{\mathcal{F}}_{k}\right)^{\frac{1}{k}}}{k}
$$

and this yields $\liminf \operatorname{in}_{k \rightarrow \infty}\left(\overline{\mathcal{F}}_{k}^{\prime}\right)^{1 / k} / k \leq \liminf _{k \rightarrow \infty}\left(\overline{\mathcal{F}}_{k}\right)^{1 / k} / k$. We deduce that $\lim \sup _{k \rightarrow \infty}\left(c\left(\overline{\mathcal{F}}_{k}{ }^{\prime}\right)^{1 / k} / k\right)^{-1} \geq \tau$.

Now, we can repeat the previous construction, starting from time $T$ and initial values $\left(c_{n}(T), \dot{c}_{n}(T)\right)_{n=1}^{\infty}$, and build a solution of the problem on $[T, T+\tau)$. This shows that $T^{*}=T^{*}+\tau$, thus $T^{*}=+\infty$. Hence Theorem 3 is proven.

\section{Damping For the multimode CASE}

We consider again the problem in $(19,20)$. This time, we assume that we have a finite number of modes $N \geq 1$. It was proven in Section 5.1 (simply using the Cauchy-Lipschitz theorem) that this problem has a solution for $t \geq 0$, moreover we have seen in Section 4.1 that if

$$
E_{\alpha}(t)=\sum_{n=1}^{N} \frac{\dot{c}_{n}^{N}(t)^{2}}{2}+\alpha \omega_{n}^{2} \frac{c_{n}^{N}(t)^{2}}{2}
$$

then $E_{\alpha}$ is decreasing, so that we have a global control of the energy.

Theorem 4. Assume for every $n, n^{\prime} \in\{1, \ldots, N\}, \omega_{n} \neq \omega_{n^{\prime}}$. Then $E_{\alpha}^{\infty}:=\lim _{t \rightarrow \infty} E_{\alpha}(t)=0$.

Proof. Consider an increasing sequence $\left(t_{k}\right)_{k \geq 1}$, with $t_{k} \rightarrow+\infty$ as $k \rightarrow+\infty$, and such that for each $n=$ $1, \ldots, N,\left(c_{n}\left(t_{k}\right), \dot{c_{n}}\left(t_{k}\right)\right)$ converge to some pair of real numbers $\left(\gamma_{n}^{0}, \gamma_{n}^{1}\right)$.

We introduce for every $k$ the functions $c_{n}^{k}(t)=c_{n}\left(t_{k}+t\right), n=1, \ldots, N$, defined for $t \geq 0$. We have for every $t \geq 0$ and $k$

$$
\frac{\mathrm{d} E_{\alpha}}{\mathrm{d} t}\left(t_{k}+t\right)=\left(\alpha-a\left(t_{k}+t\right)\right) \phi^{-1}\left(a\left(t_{k}+t\right)\right)
$$

and since $E_{\alpha}\left(t_{k}+t\right)$ goes uniformly to the constant $E_{\alpha}^{\infty}$ as $k \rightarrow \infty$ we deduce that $-d E_{\alpha} / d t\left(t_{k}+t\right) \rightarrow 0$, at least in $L^{1}(0,+\infty)$, so that $a\left(t_{k}+t\right)$ goes to $\alpha$ (a priori weakly-* in $L^{\infty}$, but in fact the results that follow will yield uniform convergence on compacts subsets of $[0,+\infty))$.

Then it is easy to show that as $k \rightarrow \infty$, the functions $c_{n}^{k}$ and $\dot{c}_{n}^{k}$ converge respectively to functions $\gamma_{n}$ and $\dot{\gamma}_{n}(t)$ uniformly on the compact intervals in $[0,+\infty)$, with $\gamma_{n}$ solving

$$
\ddot{\gamma}_{n}+\alpha \omega_{n}^{2} \gamma_{n}=0
$$

on $(0,+\infty)$, and $\gamma_{n}(0)=\gamma_{n}^{0}, \dot{\gamma}_{n}(0)=\gamma_{n}^{1}$, for every $n=1, \cdots, N$. We also get that $\sum_{n=1}^{N} \omega_{n}^{2} c_{n}^{k}(t) \dot{c}_{n}^{k}(t)$ converges (uniformly on compact sets) to $\sum_{n=1}^{N} \omega_{n}^{2} \gamma_{n}(t) \dot{\gamma}_{n}(t)$, and simultaneously to $\phi^{-1}(\alpha)=0$.

Hence, letting $P=(\alpha / 2) \sum_{n=1}^{N} \omega_{n}^{2} \gamma_{n}(t)^{2}$ and $K=(1 / 2) \sum_{n=1}^{N} \omega_{n}^{2} \dot{\gamma}_{n}(t)^{2}$, we deduce that $d P / d t=0$, so that $P$ and $K$ remain constant (since $P+K$ is a constant).

But the solution of (29) is explicit; i.e., there exist real numbers $A_{n}, B_{n}, n=1, \ldots, N$, such that for every $n, \gamma_{n}(t)=A_{n} \cos \left(\sqrt{\alpha} \omega_{n} t\right)+B_{n} \sin \left(\sqrt{\alpha} \omega_{n} t\right)$. We get

$$
\begin{aligned}
P= & \frac{\alpha}{2} \sum_{n=1}^{N} \omega_{n}^{2} \frac{A_{n}^{2}+B_{n}^{2}}{2} \\
& +\frac{\alpha}{2} \sum_{n=1}^{N} \omega_{n}^{2}\left(\frac{A_{n}^{2}-B_{n}^{2}}{2} \cos \left(2 \sqrt{\alpha} \omega_{n} t\right)+A_{n} B_{n} \sin \left(2 \sqrt{\alpha} \omega_{n} t\right)\right) .
\end{aligned}
$$


This expression is constant if and only if $A_{n}=B_{n}=0$ for every $n$, provided $\omega_{n} \neq \omega_{n^{\prime}}$ when $n \neq n^{\prime}$ (so that the $2 N$ functions $t \mapsto \cos \left(2 \sqrt{\alpha} \omega_{n} t\right)$ and $t \mapsto \sin \left(2 \sqrt{\alpha} \omega_{n} t\right), n=1, \ldots, N$ are linearly independent). Hence we have established that $E_{\alpha}(t) \rightarrow 0$ as $t \rightarrow \infty$.

This result, together with the bound in (18) proves that for the case of finite number of modes with Lipschitz control $\phi(\cdot)$, vibration from any initial condition is damped.

Remark. If $\omega_{n}=\omega_{n^{\prime}}$ for some $n \neq n^{\prime}$, the control law does not damp. A trivial counter-example is $N=2$, with $\omega_{1}=\omega_{2}=1$. A solution is $c_{1}=\cos \sqrt{\alpha} t, c_{2}=\sin \sqrt{\alpha} t$, resulting in $P=K=$ constant. Such a situation can occur if we wish to damp the wave equation in a square domain $\Omega=(0,1)^{2}$ with Dirichlet boundary conditions. The second eigenvalue $5 \pi^{2}$ is repeated and corresponds to the two eigenmodes $\sin 2 \pi x \sin \pi y$ and $\sin \pi x \sin 2 \pi y$.

Notice that a small perturbation of the system (for instance, if the domain is replaced with a rectangle $(0,1) \times(0,1 \pm \epsilon)$, for some small value of $\epsilon)$ breaks the symmetry. However, in cases where two eigenvalues are distinct but very close, numerical experiments show that the damping occurs at a very slow rate.

\section{Discussion}

We have studied a control for the wave equation where the control is a time dependent coefficient. A simple control law based on integrations-by-parts is proposed. We show that under the assumption that the vibration consists of only a finite number of modes of distinct eigenfrequencies, the control law leads to damping. In the case of one mode, the control law turns out to be optimal.

While we were able to establish well-posedness of the initial value problem for infinite number of modes, we were unable to prove damping for the system for this case. The crux of the difficulty is that we were not able to demonstrate that the energies corresponding to $\gamma_{n}(t)$ satisfying $(29)$ and the limits of the energies of $c_{n}(t)$ coincide.

Finally we remark that an interesting generalization of this problem is to consider coefficients which depend on $x$ and $t$; i.e., controllable function $a(x, t)$ (see [5] where a specific form of $a(x, t)$ is proposed). A control law similar to the one proposed in this work can be derived. Analysis of this problem would require basic theory for wave equations with time and space dependent coefficients, which unfortunately is not well-developed. However, we have performed several 1-D numerical experiments that convinced us that such a control procedure should lead to damping.

This paper was started during the first author's visit to the Institute for Mathematics and its Applications (IMA) in Minneapolis, MN, in September 2000. This visit, and a subsequent visit in April 2001, were funded in part by the IMA. Both authors express their gratitude to the IMA for making this work possible. We are also pleased to acknowledge useful conversations on this work with Jean-Pierre Puel and Stanley Osher. The first author is supported by CNRS. The work of the second author is supported in part by the National Science Foundation.

\section{REFERENCES}

[1] P. D'Ancona and S. Spagnolo, Global solvability for the degenerate Kirchhoff equation with real analytic data. Invent. Math. 108 (1992) 247-262.

[2] P. Destuynder and A. Saidi, Smart materials and flexible structures. Control Cybernet. 26 (1997) $161-205$.

[3] G. Haritos and A. Srinivasan, Smart Structures and Materials. ASME, New York, ASME, AD 24 (1991).

[4] H. Janocha, Adaptronics and Smart Structures. Springer, New York (1999).

[5] K. Lurié, Control in the coefficients of linear hyperbolic equations via spatio-temporal components, in Homogenization. World Science Publishing, River Ridge, NJ, Ser. Adv. Math. Appl. Sci. 50 (1999) 285-315.

[6] S. Pohozaev, On a class of quasilinear hyperbolic equations. Math. USSR Sbornik 25 (1975) 145-158.

[7] J. Restorff, Magnetostrictive materials and devices, in Encyclopedia of Applied Physics, Vol. 9. VCH Publishers (1994). 